\title{
LVIII. On the method of performing the simple experiment of interferences with two mirrors slightly inclined, so as to afford an experimentum crucis as to the nature of light
}

\section{R. Potter Esq.}

To cite this article: R. Potter Esq. (1840) LVIII. On the method of performing the simple experiment of interferences with two mirrors slightly inclined, so as to afford an experimentum crucis as to the nature of light, Philosophical Magazine Series 3, 16:104, 380-387, DOI: $10.1080 / 14786444008650056$

To link to this article: http://dx.doi.org/10.1080/14786444008650056

曲 Published online: 01 Jun 2009.

Submit your article to this journal $₫$

Џll Article views: 2

Q View related articles ¿ 


\section{$380 \mathrm{Mr}$. Potter on Fresnel's Experiment of Interferences}

with which they are struck will depend not so much on the velocity as the momentum of the large floating islands. The same berg is often carried away by a change of the wind and then driven back again upon the same bank, or in other cases it is made to rise and fall by the waves of the ocean, and may thus alternately strike the bottom with its whole weight, and then be lifted up again until it has deranged the superficial beds over a wide area. On these beds new and undisturbed strata may be afterwards thrown down. In other cases, when banks of mud and sand forming the top of a shoal have been made to assume various shapes by the lateral pressure of icebergs, the bed of the sea may subside, and then the disturbed beds may be overspread by horizontal strata, which may never afterwards be deranged by similar mechanical violence.

LVIII. On the Method of performing the simple Experiment of Interferences with two Mirrors slightly inclined, so as to afford an experimentum crucis as to the nature of Light. By R. PotTER, Esq.*

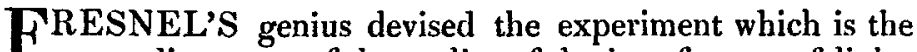
most direct test of the reality of the interference of light, and which proves that property in the most unequivocal manner. This experiment is performed by causing the light diverging from a luminous point to be reflected by two plane mirrors, placed side by side, whose surfaces are nearly in the same plane, but which contain an angle a little less than $180^{\circ}$, and then examining the light by means of an eye-lens. Each mirror gives an image of the luminous point, and we have the reflected light proceeding as if it diverged from these two images and also from its having originally constituted only one pencil, the two reflected pencils are in the same state, so that they interfere where they cross each other's direction, producing in ordinary light coloured bands parallel to the line of intersection of the planes of the two mirrors, with dark intervals between them. These bands are seen in the air in the focus of the eye-lens when looking towards the images of the luminous point.

Without examining the experiment more minutely than just to ascertain that both the pencils are necessary to the production of the bands, it must be admitted that it is conclusive in establishing the theory of interferences.

The theory of interferences was brought forward by Dr. Young as a consequence of the undulatory or wave theory of

* Communicated by the Author. 
as an experimentum crucis as to the nature of Light. 381

light; and it must be confessed that the latter theory was greatly advanced in probability by the demonstration of the former. Certain circumstances, however, such as the colour and arrangement of the bands, are required to be examined before we can consider the primary theory to be confirmed in the same extent as its subordinate theory. For instance, arcording to the fundamental property of wave interferences the central band must be white; but if it should be found in the experiment that the central band is black, this discrepancy, whilst it would not weaken the demonstration of the theory of interference, would yet be a fatal objection to the theory of undulations.

Now if two equal series of circular or spherical waves which have the same direction, or nearly so, arrive at any points in the transmitting fluid, in such a manner that the like parts of the waves arrive at the same instant, then their conjoint effect will be to produce a resultant wave stronger than either of the component waves. If, however, the two series are in a state of complete discordance, so that one series would produce an effect equal and opposite to that which would be produced by the other series, they would counteract each other, that is, no resultant wave would be produced. Two series of waves, which are respectively of equal diameters, and similar, at every instant may be denuminated simultaneous; and if we draw a line bisecting perpendicularly that joining their origins, the waves of each series will meet in the same state everywhere along this line, since every point in it is equally distant from the two origins. When these waves are very distant from the origins compared with the distance of the origins from each other, they will have very nearly the same directions, and we shall have resultant waves of greater strength than the component waves. This is a conclusion depending only on the fundamental properties of waves, and does not involve any review of the various hypotheses which are held, as to the nature of the vibrations of the particles of the fluid through which the waves are propagated.

The experiment with the two mirrors, before mentioned, produces the case we have just discussed; for the single pencil diverging from the original luminous point, is made into two pencils after reflection by the mirrors; and these two, if light consist of waves, are composed of waves both equal and simultaneous. We have here then an experiment on which to test the wave theory of light, and the experiment itself shows us which is the band corresponding to the line bisecting perpendicularly that which joins the luminous images of the point. For if the luminous point be formed of white light, 


\section{Mr. Potter on Fresnel's Experiment of Interferences}

the various colours having different intervals for their luminiferous surfaces or different lengths of waves in the undulatory theory of light, the bands will be of different breadths for different colours, but will have the one on the bisecting line above named bright for every colour, and which will be consequently white, when the luminous point is formed with white light; but on each side of it, the superposition of bands of different breadths corresponding to different colours, will cause the compound bands to be coloured, at first on their edges only; afterwards the colours will become more and more spread, and the bands at the same time more confused, as the distance from the central band becomes greater, until they are at length gradually lost in a light uniform to the eye. The bands on each side and near to the central one will have their inner edges violet and their outer edges red, so that the arrangement of colours will be symmetrical on each side of the central white band. The central band is thus pointed out in the experiment by the arrangement of the colours.

In my early trial of this experiment, I happened to have a clear sky and unclouded sun, which afforded a result causing me to hesitate before I accepted the undulatory theory as true. I have seen the experiment on days in which there were thin clouds in the atmosphere, and once when the sun was near the horizon, such that it would have led me to a different conclusion.

In my first experiments I was surprised to find the colours symmetrical on each side of a dark band, and not a bright one. Every precaution was used, such as keeping the bands clear of the diffracted fringes formed by the edges of the mirrors, making the distance between the luminous images so small that the bands were very large, and therefore that any prismatic effect produced by slight error of looking centrically through the eye-lens did not produce a sensible effect in the arrangement of the colours; also care was taken that the direct rays (as the term direct is used in optics in contradistinction to oblique) passing through the lens forming the luminous point were those which fell on the two mirrors. Still the central band was a dark one and not a bright one. I obtained the assistance of friends accustomed to accurate observation, to examine the appearances, and they came to the same conclusion. I was thus at a loss to conceive how the advocates of the undulatory theory could state that the central band was always a white one. Some time afterwards, however, I obtained a different result; for experimenting one fine evening when the sun was near the horizon, I saw the middle band clearly white, and the colours accurately symme- 
as an experimentum crucis as to the nature of Light. 383

trical on each side of it, although the whole appeared misty and without that darkness in the intervals which $I$ had found in the previous experiments. This continued whatever pains I took to keep every part of the apparatus in adjustment. The dullness and mistiness of the latter phænomenon led me to conclude that there could be no doubt but the former was the normal result. I however tried the experiment again with every care in the former circumstances, and found the same result as formerly.

In the Number of Phil. Mag. for April, 1833, p. 279, I stated, "'The result of considerable experience with me is, that it may be seen both black and white, though with me it has much oftener been the former, especially when the bands have been well defined," \&c. \&c.

In the November Number for 1833, p. 342, I said, "I shall consider it extremely important to determine whether, when an achromatic lens of short focus is used to form the luminous point, the central band of direct interference given by two mirrors is black, as it has appeared to me, and to several friends to whom I have shown it, when adequately tried with a common lens," \&c., \&c.

I have in the papers from which the above extracts are taken, publicly stated my difficulties without reserve, and my ideas of the requirements for a decisive mode of experimenting.

A mode of experimenting has been adopted, (where or with whom the discovery originated I do not know,) which is stated to give the central band white. It is this: the image of the sun formed in the focus of a lens is made to fall on a small aperture in a thin plate of metal; the light passing through the aperture falls on the two mirrors, and the aperture thus illuminated is called the luminous origin. I am informed, that when the rays falling on the two mirrors are those which pass obliquely through the aperture, then the central band is most distinctly a white one; and it has been argued that this must be the normal way of trying the experiment, inasmuch as the rays of all colours will have accurately a common origin on the edge of the aperture.

It has been with me a subject of frequent study to find out a method of trying the experiment which must give a result not to be disputed; and I feel confident the apparatus described in this paper fulfils every desideratum. The points kept in view have been to employ only such parts as are essential in the simplest form of the experiment; and thus in dispensing with the mirror which is 


\section{Mr. Potter on Fresnel's Experiment of Interferences}

usually employed to throw the sun's light through the window-shutter into the darkened room, I have employed an equatorial method of mounting, which keeps all the apparatus in the adjustment it is first placed in, and at the same time enables us to follow readily the sun's daily motion, keeping at the same time the room quite dark.

To obviate all objections which attach to the luminous point being formed by a common lens, which has different foci for differently coloured rays, I have employed a spherical mirror, as better even than any achromatic lens; and again, as it was suggested to me that an objection might be raised if the rays crossed in a real focus, I have used a convex mirror, so that they diverge from a virtual focus without having crossed : also to enable me to use the luminous point the smaller, I have generally used the two mirrors slightly inclined, of polished speculum metal, which reflects many times the quantity of light which glass reflectors do in the position which my apparatus requires. With these precautions, I find, when the sun is perfectly unclouded, and near the meridian, high above the horizon, that the central band is black. When there are clouds before the sun's disc, however slight, the central band is more difficult to $f \mathbf{x}$ upon, and generally either white or doubtful. The discrepancies which have been to me a puzzle for so many years, I am now able to solve, and I announce the following new principle of interferences :-

When light in a state of interference is made to interfere again, the result is of an opposite character to what it would have been if the light had been in the first instance in the ordinary state.

This proposition, which is in itself reasonable, might have been anticipated, and solves all the anomalies. The light falling on the small hole in the thin plate of metal, formerly mentioned, as used to form the luminous point, is thrown into a state of interference by diffraction at the edge of the hole, and hence the central band is seen white. Again, the sun's light passing through thin clouds, or through the vatpours of the atmosphere when near the horizon, is thrown more or less into a state of interference by diffraction at the edges of the particles of the vapours, and gives results which are either doubtful or with a white centre: for where part of the light is in its original state and part in a state of interference, the bright and dark centred bands will be superposed, the central band of the one set over the central band of the other, and thus produce indistinct phænomena in which the intensity of the one or the other species may prevail. 
as an experimentum crucis as to the nature of Light. 385

From the full investigation, I thus maintain that the normal result of this fundamental experiment in interferences, is, that the central band is black, and at variance with the consequences of all wave theories of light, and therefore the undulatory theory of light is not the physical theory.

This conclusion is also borne out by the phænornena of the rainbow, (see Lon. and Edin. Phil. Mag, for July 1838, vol. xiii. p. 9.) the actual primary and secondary bows being much more distant than they should be from all calculations according to the undulatory theory of light, the position calculated by that theory to be bright being actually found to be dark. In the artificial methods of producing the analogous effect with a minute stream of water, there is no doubt but that the effect calculated from the undulatory theory might be obtained, by using a line of light in a previous state of interference.

The apparatus is represented in figs. 1 and 2, in which the

Fig. 1.

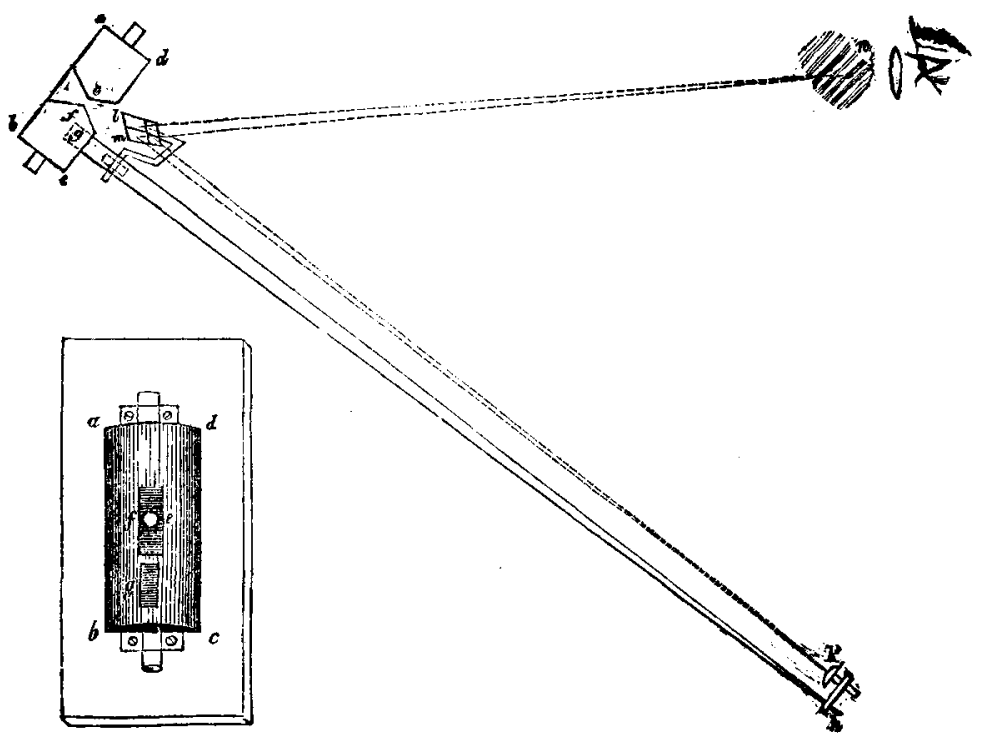

Fig. 2.

same letters refer to the same parts : $a, b, c, d$ is a cylinder of wood about three inches in diameter, and seven inches in length from $a$ to $b$, with pivots as in the figures. In fig. 2 this cylinder is seen placed in a rectangular aperture cut in a thick piece of board in which it exactly fits, and is retained in 


\section{$386 \mathrm{Mr}$. Potter on Fresnel's Experiment of Interferences}

its place by steps screwed over the pivots. An aperture is cut straight through the centre of the cylinder, which in the axis at $e f$ is circular, of a little more than half-inch diameter, but towards the surface it is extended longitudinally: through it the sun's light falls on the small convex mirror $k$. This mirror is attached, as in the figure, to the $\operatorname{arm} g h$, made of light wood, about four feet long, which fits tightly in a lateral direction in a groove $g$, fig. 2 , but can be moved in a plane passing through it and the axis of the cylinder, so as to place the mirror in the sun's light shining through the hole. The axis of the cylinder is fixed parallel to the earth's axis, when the apparatus is finally secured in the window-shutter of a room with a south aspect. The form of the aperture allows for the sun's declination at different times of the year, by which means, and the motion of the arm, the sun's light can be made to fall on the mirror $k$ at all seasons; and to follow the sun's daily motion we have only to push the arm so as to turn the cylinder about its pivots. Every part is made to fit just so tight that the apparatus remains in any position in which it is placed. The two mirrors are placed as at $l \mathrm{~m}$, so that the sun's light just passes their edges when it falls on the mirror $k$; by this arrangement we make it certain that the light received by the two mirrors is that which is reflected nearly directly by the mirror $k$, and therefore with very little aberration. The two mirrors are attached to a piece of wood, which, having a hole in the line of contact of the mirrors, is moveable about a thick wire which fits tightly into this hole. This piece of thick wire is bent at right angles in three places, as seen in fig. $l$, and forms a very convenient universal joint, which allows the mirrors to be turned about in any direction required: at its lower part it is pushed through a hole in a piece of wood fixed to the side of the arm $g h$. The dotted lines $n l \mathrm{mk}$, are intended to show the course of two rays from the mirror to their interference at $n$ in the focus of the eye-lens.

All the parts of the apparatus should be well blackened, and a piece of black velvet (as the most perfect black) placed behind and about the mirror. It is also desirable to have a tube of blackened paper placed on the arm near $k$, through which the sun's light may shine, but which will prevent the stray light from the mirror $k$ injuring the darkness of the room.

Those who understand analytical geometry will find the readiest way of fixing the cylinder parallel to the earth's axis, to be that of calculating the lines of intersection of the board in fig. 2 with the plane of their window-shutter: great accu- 
racy is not needed in fixing the cylinder, as its use is merely to afford the means of following the sun's daily motion and still to leave the room dark.

As the apparatus is here drawn, it will be advisable to have the mirrors $m l$ of speculum metal, for the angle of incidence of the light is too small for glass mirrors to give very bright phænomena; although I have seen them of the same character as with the mirrors of speculum metal. The mirror $k \mathbf{I}$ have used of one-fourth of an inch focus.

All the mirrors should be of metal which is not porous, and of the highest polish, so that their surface is not visible when they are placed a short distance from the flame of a candle; for it is probable that numerous small pores in the metal, or the fine lines which constitute a second-rate polish, would produce diffraction sufficient to invalidate the experiment. As such mirrors are not everywhere to be had, I should, in such cases, recommend, as a substitute, that a good lens, of short focus, well centred, be placed in the aperture $e f$, and that a pair of glass mirrors blackened at their second surface, be placed on the arm at the other end $h:$ the incidence would then be sufficiently oblique to afford an intense reflexion. Ordinary plate glass, or good pieces of window-glass, will show the bands very well; but those who wish to test the colour of the central band, should have their mirrors, as well as their lens, of good workmanship. The obtuse-angled prisms which may be obtained in the shops, are very convenient for observing the bands popularly, but are not to be depended upon as test apparatus.

Those who use the two mirrors for the first time will find some care necessary in fixing them with their contiguous edges in contact, for if the edge of one be raised any appreciable distance above that of the other, the bands are not produced. With glass mirrors, blackened at their second surface by sealing-wax melted over them, it is convenient to cement them together with the same material along their line of contact. If they are placed in position whilst the sealingwax is soft, and then retained in their position until cool, they will remain ready adjusted to be used at any time.

I have been the more minute and popular in explaining the theoretical bearings, as well as the experimental details, from the consideration that this interesting and important experiment has very singularly been but little noticed in our more descriptive optical treatises; and also from the hope that I may by this essay induce others to try it, who have a sincere wish to form a correct opinion on the nature of light. 